\title{
Décadrages Décadrages
}

cınéma, a travers champs Cinéma, à travers champs

12 | 2008

Fredi M. Murer

\section{A propos du «cinéma privé » de Fredi Melchior Murer}

Marcel Leiser

\section{OpenEdition}

Journals

Édition électronique

URL : http://journals.openedition.org/decadrages/348

DOI : $10.4000 /$ decadrages.348

ISSN : 2297-5977

Éditeur

Association Décadrages

Édition imprimée

Date de publication : 10 avril 2008

Pagination : 11-20

ISBN : 978-2-9700582-7-4

ISSN : 2235-7823

Référence électronique

Marcel Leiser, «A propos du « cinéma privé » de Fredi Melchior Murer », Décadrages [En ligne],

12 | 2008, mis en ligne le 10 avril 2009, consulté le 22 avril 2019. URL : http://journals.openedition.org/ decadrages/348; DOI : 10.4000/decadrages.348

(B) Décadrages 


\title{
A propos du "cinéma privé"
}

\author{
de Fredi Melchior Murer
}

par Marcel Leiser 1

"Dans le monde humain, les régions périphériques me sont plus familières que le milieu.»

Fredi M. Murer

"Sans pensée alternative, il n'y aura pas de siècle prochain.»

Max Frisch
1 Marcel Leiser signe ici le témoignage "ludique " et "non archéologique " d'une époque et d'un milieu où il fut aussi un acteur actif: fondateur et rédacteur en chef de la revue Travelling en 1963, cinéaste - Les Trois regards, 1967, Une fille et un fusil, 1969, Nathalie ciné-roman, 1970, Le bonheur à 70 ans, 1974, La dame de la véranda, 1974, Bo-go-tà, 1976 - et journaliste, il mit notamment sur pied en 1968 à Lausanne une structure de diffusion alternative, Cinéma Marginal Distribution.

2 Avant de réaliser des films $35 \mathrm{~mm}$ intégrés dans le circuit commercial, Murer a pratiqué, selon sa propre expression, du "cinéma privé", le plus souvent des films-portraits $16 \mathrm{~mm}$ d'amis de la scène artistique underground, dans un esprit post-surréaliste, voire expérimental, sans motivations sociales ou politiques: des œuvres destinées d'abord à ses proches, sans souci de toucher un public élargi de salles de cinéma conventionnelles. De son premier court métrage 8mm, Marcel, en 1962, jusqu'à Christopher \& Alexander, en 1973, il défend un cinéma libertaire à très petit budget, sans scénarios élaborés, sans "vrais" acteurs et privilégie l'improvisation au tournage. Pour produire, il disait, avec humour, être son propre mécène, mais, la reconnaissance arrivant, il bénéficia tout de même du mécénat de la Banque populaire suisse pour Swissmade-2069 (1969), puis du banquier Eric Franck pour Christopher \& Alexander. En 1974, Wir Bergler in den Bergen sind eigentlich nicht schuld, dass wir da sind, documentaire tourné avec des Uranais rencontrés lors des funérailles de son père, lui fera quitter la bulle juvénile de l'underground pour s'orienter vers une œuvre davantage inspirée par la société dans sa globalité.
Flash-back: Soleure 66, première! Fin janvier 1966, la Guilde du Film de Soleure (un ciné-club comportant pas moins de mille membres) organise 

une nouvelle génération de cinéastes ancrés dans la modernité, refusant ou ignorant la tradition du Heimatfilm et de l'idéologie horlogéro-fromagère. Apparaît aussi le débat, souvent vif. Alors que le froid hivernal et nocturne recouvre la ville, la température monte au Chutz, un restaurant moderne où, sur deux étages bourrés de cinéphiles passionnés, s'affrontent positions et propositions : une grande discussion divise les tenants d'un cinéma "populaire et de qualité » (déjà...) à ceux qui prônent les films d'art et d'essai sans concessions. Une chose est sûre: le jeune cinéma suisse veut exister, coûte que coûte. Avec énergie, passions et colères.

Deux réalisateurs s'opposent au Chutz avec vivacité, mais courtoisie. Niklaus Gessner, qui a montré Un milliard dans un billard (1965) (sympathique premier long métrage $35 \mathrm{~mm}$, une comédie policière avec Jean Seberg et Claude Rich), ne croit qu'à l'avenir d'un cinéma commercial, divertissant et avec vedettes, seul susceptible de connaître une large diffusion internationale. Au contraire, Alexander J. Seiler, qui a présenté Siamo Italiani (1964) (son premier long aussi, un remarquable documentaire en noir-et-blanc sur l'immigration ouvrière), défend sérieusement et ardemment un cinéma d'auteur, quitte à ce qu'il dérange les normes commerciales routinières. Un cinéma qui ouvre les yeux sur la réalité et contribue à développer une identité nationale rénovée, grâce à une réflexion critique.

Fredi Melchior Murer est présent lors de ces premières Journées soleuroises avec Pazifik - oder die Zufriedenen (1966), bande d'une heure tournée en muet et sonorisée après coup. Découverte d'un poète de l'underground qui est, bien sûr, du côté de l'art et essai (dans ses marges plutôt), mais fort loin des "professionnels de la profession". D’ailleurs son film a failli être refusé et il a fallu l'insistance appuyée de son épouse auprès des sélectionneurs pour qu'il figure au programme! Rendant compte de "Soleure 1», j'écrirai, de retour en Romandie: "C'est une réinvention délirante du cinéma, pleine de superflu et de gratuité, mais contenant plusieurs séquences oniriques d'un lyrisme authentique»3. C'est, effectivement, la révélation d'une nouvelle voie ici, celle d'un art qui ne se préoccupe pas des conventions du genre. Qui invente visuellement. Un éclat de fantaisie poétique surgissant dans un environnement formaté. Fredi Murer crédibilise l'idée que l'on peut faire du cinéma libre s'affranchissant des lourdeurs traditionnelles, et il devient, dès "Soleure 1", un phare du cinéma de la périphérie. En marge des caméras-tanks, les caméras-stylos montent au front...

Au fil des ans, je retrouverai régulièrement Fredi à Soleure et ailleurs. Contact un peu timide, mais amical: il n'aime guère parler français et 
je peine avec l'allemand, mais nous échangeons à chaque fois quelques mots cordiaux, sans grands discours. En 1969, juste avant la sortie de Swissmade, je lui rends aussi visite dans son atelier zurichois du Zeltweg. C'est dans cette même rue, au sous-sol du $n^{\circ} 86$, que, quelques décennies plus tôt, Tristan Tzara composa Dada 3 chez son imprimeur préféré, l'anarchiste Julius Heuberger. De Dada au jeune Murer, l'esprit frondeur s'est ici perpétué, envers et contre tout.

Au Zeltweg, je découvre une petite bicoque qui ne ressemble en rien à un bureau de réalisateur de films "overground". Au cœur d'une cité qui s'est modernisée, cela a plutôt l'air d'une cabane où un gosse jouant aux Indiens s'inventerait un monde imaginaire que personne ne lui impose. Les Indiens sont encore loin à la Bahnhofstrasse, mais peut-être viendront-ils fumer le calumet de la paix dans ce refuge avec une verrière qui ferait aussi penser au studio - certes en plus petit - de Georges Méliès, rempli de maquettes, de dessins et de bricolages. Méliès, Murer, deux voyageurs vers la lune qui savent connecter poétiquement l'image avec l'imaginaire. L'univers de Fredi, c'est d'abord la présence du monde de l'enfance qu'il refuse de quitter et qu'il prolongera même au travers de plusieurs personnages de ses longs métrages. A ce momentlà, il me fait penser à un garçon qui joue aux allumettes de Bengale pour en faire jaillir des feux d'artifice, comme si leurs couleurs et leurs soudaines et éphémères lumières pouvaient annoncer l'aurore. Ou à un extra-terrestre un peu égaré dans la bien-pensante et repliée Confédération helvétique.

Bien qu'artiste marginal, le cinéaste est devenu une figure incontournable de la "famille» du cinéma suisse. Une famille toute neuve, dans laquelle jeunes menant un vie de bohême et Doktoren se côtoient dans une solidarité certaine, en Suisse alémanique particulièrement. Murer, le visage rond comme la lune de Méliès (forme pacifique, en signe de non-violence), des yeux observateurs et curieux s'ouvrant aussi sur un monde intérieur nocturne et étoilé, des lèvres exprimant humour et amertume, apparaît plutôt discret et calme (c'est un taiseux... proche du jeune héros de Höhenfeuer (1985) qui, lui, est carrément un muet). Mais, quand il prend la parole, sans s'exciter, Murer distille alors une ironie décapante, ignorant clichés et dogmes. Une voix singulière, débordant d'imagination, et d'une drôlerie magicienne. Il n'y a pas de dichotomie entre l'homme et son œuvre.

\section{Le temps de la folie débridée}

Flash-back bis: Zurich City. Pour qu'un cinéma créatif existe, il faut un terreau, un vivier de rêveurs, des rencontres. Rien de mieux qu'une 
4 Fredi M. Murer, Collectif, Pro Helvetia, Zurich, 1980 , p. 7.

$5 \mathrm{lbid}$.

$6 / d .$, p. 15. grande ville centripète où se retrouvent artistes, freaks et autres inventeurs d'utopies. A ce titre, Zurich apparaît sans doute comme la seule vraie capitale culturelle et artistique de Suisse, et ceci à diverses époques. Comme au temps de Dada et du cabaret Voltaire. Comme au temps du nazisme qui engendra un afflux d'immigrés, temporaires ou permanents, fuyant l'Allemagne et l'Autriche, gens de théâtre, de cinéma, de musique, de littérature. Comme dans les années 1960 où l'on imagine Frisch et Dürrenmatt attablés au café Odéon, stamm où l'on se retrouvait entre "révolutionnaires culturels». C'est dans cette vénérable ville de Zurich que Fredi Melchior, venu de sa campagne de carte postale, commence à manier une petite caméra, tout émoustillé.

Fils d'un menuisier et d'une couturière, petit-fils d'une femme qui composait des poèmes et lui chantait des Lieder, Fredi Melchior Murer voit le jour en 1940 en Suisse centrale au bord du Lac des QuatreCantons (canton de Nidwald) et grandit dans le canton d'Uri. Autres temps, autres coutumes, il n'est pas «biberonné» au cinéma dès sa petite enfance: il est déjà vieux de treize printemps lorsqu'il voit le premier film de sa vie, Le Kid (The Kid, 1921), de Chaplin. "Au cours des semaines suivantes, je m'identifiais tellement à Jackie Coogan que j'en oubliais même d'aller à l'école» raconte-t-il4. A 16 ans, des dessins sous le bras, il quitte précipitamment école et famille pour «monter" à Zurich, où il cherche à pratiquer "un métier dont l'exercice ne rendrait service à personne sauf à moi-même»5. A 19 ans, il finit par entrer à l'Ecole des Arts Décoratifs de Zurich où, après un an de dessin technique, il choisit d'entrer en classe professionnelle de photographie. Lors d'une exposition, consacrée au Septième art et montée dans l'Ecole même, les étudiants sont conviés à photographier, séquence par séquence, des films de Flaherty, Dreyer, Buñuel, Vigo et d'autres. Une révélation!

Murer s'achète alors une petite caméra et se met à filmer en parfait autodidacte: "C'était sortir du silence par le silence: dans ses premiers films on ne parlait pas " constate Richard Dindo ${ }^{6}$. Le cinéaste en herbe vit alors dans une vieille villa de la Plattenstrasse 47, avec une bande de copains non-conformistes et idéalistes qui croient qu'une alternative à la société dominante est possible et que l'on peut changer la vie dans un esprit festif, en installant l'imagination au pouvoir, sans barrière entre le rêve et la réalité. C'est dans ce décor communautaire qu'est tourné le film-clé génésiaque du jeune cinéma underground suisse, Pazifik-oder die Zufriedenen, de 1962 à 1965. D’une durée de quatre heures, il est réduit à une heure pour "Soleure 1 ", et deux épisodes sortiront après-coup sous forme de courts métrages, Balance et Sylvan. 
"Murer au début, analyse Richard Dindo, fait des films de famille, mais ici la famille ce sont des copains, des gens comme lui, ce sont eux ses premiers personnages et ils sont aussi les premiers spectateurs. Ça se passe dans une société à part qui n'a rien à faire avec celles des autres, on est loin de 68, on est loin de la politique. Il faut comprendre ses premiers films comme un refus de tout ce qui pourrait venir de la génération des pères, comme un refus d’apprendre d'eux, de compter sur eux.» $\mathbf{7}$

Dans ce petit monde marginal, graphistes, photographes, musiciens et artistes en tous genres voient déjà en lui une figure de proue: le King Melchior of Cinema 8 !

Il y a la "famille» de la Plattenstrasse, mais des liens se sont aussi tissés avec d'autres milieux zurichois. Le jeune Fredi fait partie de la Zürcher Wabnwelt ("monde de l'imaginaire»), un cercle d'artistes zurichois, peintres et écrivains qui veulent en découdre avec la désormais stérile Suisse d'antan. Parmi les membres, l'on se souvient en particulier de l'écrivain Paul Nizon, du peintre Friedrich Kuhn, du cinéaste Georg Radanowicz, ainsi que de plusieurs personnages qui "animent» les premiers films de Murer : le poète du Kellertheater Urban Gwerder, au look de Salvador Dali (Chicorée, 1966), le peintre (et marcheur) Alex Sadkowsky (Sad-is-Fiction, 1969) et le plasticien Hans-Ruedi Giger (Passagen, 1972), bien avant ses décors d'Alien (Ridley Scott, 1979). Un milieu surréalisant et libertaire, doté d'un enthousiasme jubilatoire. Dans l'étouffante gérontocratie helvétique, une jeunesse déjantée tente ainsi de prendre le pouvoir, ou, pour le moins, d'exister. Pour le plaisir sans doute, mais en semant tout de même quelques graines pouvant générer une civilisation où l'on pourrait mieux souffler: "Faites usage de la liberté de pensée et de parole, avant qu'elle ne soit atteinte par la rouille» $\mathbf{9}$.

En 1966, l'année de "Soleure 1", le cinéma en liberté et de format sub-standard connaît un début d'organisation. Entouré d'une bande de jeunes cinéastes débutants, telle une génération spontanée, Hansjakob Siber ${ }^{\mathbf{1 0}}$, dit Köbi, visage grave, tignasse christique et caractère un peu rocailleux, met sur pied à Zurich une coopérative sur le modèle du New American Cinema, Filmforum: à la fois un ciné-club, une petite coopérative de production et de distribution, ainsi qu'un programme itinérant de films qui circule dans les villes alémaniques sous le nom de Ciné-Zirkus. "Ce cinéma différent (das andere Kino) est devenu une réalité vivante en Suisse alémanique»11. Et, complice des activités de Köbi, Murer lui confie plusieurs de ses films, en particulier Meteorit et Balance 12. Tout au début des années 1970 (et comme Cinéma Marginal Distribution à Lausanne), Filmforum cèdera le terrain à un organisme
$7 \mathrm{lbid}$.

8 Surnom que lui donnaient ses amis, mentionné dans un article d'Alexander J. Seiler dans le Zürcher Almanach, en 1968, et repris dans Fredi M. Murer, op. cit., p. 59.

9 Max Frisch, Discours du $1^{\text {er }}$ août, Zurich, 1957.

10 Né en 1942, Siber, après une année d'études de théâtre et de philosophie aux EtatsUnis, devient fondateur-animateur de Filmforum (qui avait son siège à la Stotzgasse 60 , à Zurich). Siber était aussi réalisateur de nombreux films underground, très influencé par les pellicules informelles du New American Cinema. Citons La Légende du vieux Berger Firmin et de son ami Reimann (16mm, 60', 1974), une belle œuvre tardive, au moment où il se consacrait professionnellement à une autre passion: la recherche, la collection et la détermination de minéraux.

11 Marcel Leiser, Journal de Genève, Genève, 18.01.1969.

12 Ces deux courts métrages furent extraits en 1967 de Pazifik. Cf. filmographie de Murer parue dans Travelling, $\mathrm{n}^{\circ} 22$, p. 23. 
13 Marcel Leiser, Radio-TV - Je vois tout, janvier 1968. Texte écrit sous le pseudonyme d'Alexis Duval. de diffusion «officiel» et subventionné, le Film-Pool du Centre suisse du cinéma. Début de la fin d'une époque euphorique et débridée, et place au développement d'un cinéma plus formaté professionnellement... et surtout plus conforme aux standards souhaités par la télévision du prime time!

\section{L'internationale de "l'underground " fait la fête}

Flash-back ter: Knokke 67-68, dernière! Fondée en 1949, la Compétition internationale du film expérimental vit sa quatrième (et dernière) édition à Knokke-le-Zoute fin décembre 1967 et tout début 1968, orchestrée sans vedettariat par Jacques Ledoux, le très curieux directeur de la Cinémathèque royale de Belgique, à l'apparence d'un Tournesol de sciencefiction. Un moment historique pour tout le milieu de l'underground mondial, et qui ne sera jamais renouvelé, apparemment parce que le président de la Cinémathèque, un ponte de la Justice belge, n'a guère apprécié l'esprit libertaire potache, voire licencieux (avec, sous l'esprit, des nudités de tous sexes confondus) qui a plané cet hiver-là sur la grise station balnéaire de la Mer du Nord. Pour mémoire, quelques phrases tirées d'un des articles rédigés à mon retour de Belgique:

«Knokke-le-Zoute a été le festival du cinéma en délire... Nombres d'œuvres expérimentales se distinguaient par un refus des techniques traditionnelles de narration cinématographique. Ecrire un scénario avant de tourner un film est considéré généralement par les 〈expérimentalistes〉 comme un acte sacrilège privant l'artiste de sa spontanéité et de sa liberté. Donc pas de scénario, mais un cinéma dit de poésie... Cette expression tient aussi à s'interdire tout ce qui pourrait s'apparenter à un art appelé bourgeois.»13

Ambiance festive s'il en est, le spectacle se vit à Knokke autant dans la salle de projection du Casino qu'en dehors. Ce cinéma off d'une liberté déchaînée s'offre même des manifestations "off off». Avec son chapeau mou masculin toujours vissé sur le chef, Shirley Clarke, l'une des figures de proue du New American Cinema, organise des séances sauvages avec son petit projecteur $16 \mathrm{~mm}$ partout où il y a une prise électrique (encore faut-il la trouver!). Avec quelques happy feres, je visionne ainsi des bandes très underground, en particulier dans le couloir de l'hôtel de la cinéaste, juste en face de sa chambre. En concours, c'est un jeune New-Yorkais inconnu au bataillon, un certain Martin Scorsese, qui remporte le Prix de l'Age d'Or avec The Big Shave (1967). L'impertinente Yoko Ono, venue de la scène underground londonienne, montre en compétition Film Number 4 (1966), où elle filme 365 postérieurs marchant - en gros plan - pendant 80 minutes. Elle se distinguera aussi (et entre autres) en 
participant nue à l'élection-bidon de Miss Cinéma Expérimental, un happening organisé par le Français Jean-Jacques Lebel, grand spécialiste du genre. Sur le grand écran, un Anglais, Stephen Dwoskin14, primé ici pour trois courts métrages, confirme la crédibilité et l'importance d'un cinéma différent, en filmant des images envoûtantes de jeunes femmes. Le mystère féminin en pellicule gros grain!

Présent déjà à Knokke-le-Zoute lors d'une précédente édition où il avait découvert, enthousiaste, un film de six ou sept heures d'Andy Warhol, «King Melchior» se sent, à l'évidence, sur la même longueur d'ondes que les autres artistes multi-disciplinaires membres de cette internationale libre de la création périphérique. Pour lui aussi, le cinéma est une fête! Et d'affirmer, péremptoire :

"Mes visites aux festivals du film expérimental de Knokke ont bien

plus vivifié les circonvolutions cinématographiques de mon cerveau

que tous les classiques réunis. »15

A ses côtés, je retrouve, dans le hall du Casino, la modeste «délégation » suisse. Je me souviens, en particulier, de Köbi Siber qui montre Jalousie, gratté sur pellicule, sans caméra. De Hans Helmut Klaus Schönherr 16, le réalisateur expérimental le plus jusqu'au-boutiste travaillant en Suisse; il propose ici Thaler's, Meier's, Sadkowsky's Life in the Evening et prépare un film consacré à Robert Walser, figure alors emblématique de tous les Neinsager 17. De Georg Radanowicz, un cinéaste-auteur aujourd'hui trop méconnu, représentant à Knokke le Zürcher Wabnwelt avec le burlesque Pic Nic. De Martin Schaub enfin, brillant critique zurichois et fin analyste du cinéma suisse, accompagné de sa belle maîtresse en robe longue coulant jusqu'aux pieds. Sans oublier, forcément, la joyeuse bande d'AKS Film (Aebersold, Klopfenstein et Schaad) qui donne à voir Wir sterben vor.

Un cinéma hors-sytème est donc possible et existe. Issu, avant tout, des actions des gens du milieu des arts plastiques, pour qui le Septième art amène, en sus de leur premier moyen d'expression visuel, le mouvement. Les festivaliers suisses prennent conscience à Knokke-le-Zoute de leur appartenance à cette internationale libertaire, celle de l'opposition individualiste, plus subversive qu'elle n'en a l'air. Malgré les barrières alpines, les hiérarchies obsolètes et l'esprit d'enfermement de la mèrepatrie, ils réussissent à pratiquer l'ouverture en tous sens, prenant part, eux aussi, aux nouvelles énergies de cette planète qui est en train de bouger. Et ils s'extirpent de leur réduit à croix blanche sans faire de complexes! Car il est temps de raser les montagnes du conformisme fotal suisse pour mieux voir les vastes horizons de l'avenir libéré. D'inventer des îles d'expression artistique où souffle le vent du large, et cela peut
14 Sur Stephen Dwoskin, voir le dossier consacré par Décadrages, nº 7, printemps 2006.

15 Dokumentarfilme aus der Schweiz, Kellerkino, Berne, 1977, et repris dans Fredi M. Murer, op. cit., p. 52.

16 Né en 1936 en Allemagne, HHK Schönherr a suivi les cours de la Hochschule für bildende Künste à Hambourg, avant d'étudier et de s'établir à Zurich. Peintre, ami de cinéastes de l'internationale de l'underground comme Gregory Markopoulos ou Robert Beavers, il est devenu, sans aucun doute, le plus radical des avantgardistes du cinéma suisse. Parmi ses films, citons Autoportrait (16mm, 120', 1968), sa série Play 1, Play 2, Play 3, etc. et Das Kaputte Kino (35mm, 12', 1971).

17 Très usité par les intellectuels de gauche des années 1960, le terme de Neinsager (celui qui dit non) désignait alors tous ceux qui contestaient, passivement ou activement, l'ordre établi conservateur: intellectuels, artistes, politiques, marginaux, anarchistes, voire même malades mentaux. Depuis le début des années 1990, ce vocable définirait plutôt les récriminateurs de la frange conservatrice et réactionnaire de l'échiquier politique. 
18 "Experimentalfilm», in Cinema, $n^{\circ}$ 68, Zurich, 1972, p. 1116. commencer dans des caves ou des arrière-salles de bistros, de Zurich ou Lausanne: le patriotisme fromager, basta! Ailleurs comme ici, ce souffle nouveau apporte une réponse circonstancielle au monde dépassé des "pères", la société doit changer de cap. En effet, l'Amérique bombarde le Vietnam, là-bas, alors que la gérontocratique et militairement hiérarchisée Helvétie survit ici dans un protectionnisme stérile hérité de 193945. Et, partout, la civilisation occidentale mise sur la surconsommation devenue philosophie de vie.

De nombreux jeunes, les artistes en première ligne, s'inventent des espaces de création et répandent un non-conformisme libérateur. Cette opposition individualiste sera, peut-on supposer, plus créatrice pour un changement des mentalités que le militantisme politique spontané ou reposant sur des modèles dogmatiques d'antan. Murer fait évidemment partie de cette vague individualiste et non politique bien visible à Knokke-le-Zoute. Plus tard, Schönherr confirmera lui aussi, lors d'un coup de fil à Xandi (c'est le petit nom d'Alexander J. Seiler), l'importance de cette manifestation expérimentale pour son propre "cinéma privé»: "J'étais complètement surpris qu'il y avait là des gens qui pensaient d'une façon semblable à la mienne»18, un propos corroborant le point de vue de Murer.

Ces énergies inspiratrices éclatent dans les marges, psychédélisme y compris. Dans ce bouillonnement de la contre-culture, peintres, graphistes, théâtreux, poètes, écrivains, photographes, cinéastes font table rase de la pesante tradition, advienne que pourra. Et surtout les musiciens : c'est le temps du Velvet Underground, de Janis Joplin, Bob Dylan, Joan Baez, Jimi Hendrix, des Rolling Stones et de Pink Floyd... Les faiseurs de films de Knokke font partie de ce même mouvement venu de la périphérie. Si le pop-rock deviendra populaire, le cinéma underground restera, lui, confiné à un public plus confidentiel. A méditer! Et même Murer, grand défenseur du "cinéma privé», entrera dans le système commercial pour poursuivre son œuvre et fera des films à scénarios écrits. En tout bien, tout honneur d'ailleurs.

\section{Energies marginales, expressions libres}

Des images avant toutes choses, et un peu de technique! Le jeune cinéma suisse des années 1960-1970 qui se fait connaître internationalement en salles art et essai (en particulier celui réalisé par le Groupe 5, de Genève) se distingue, au départ, par un style d'écriture qui repose sur une technique nouvelle, le tournage $16 \mathrm{~mm}$ en son direct, avec des caméras silencieuses prévues à l'origine pour la télé (dont la fameuse EclairCoutant), la pellicule étant, au final, gonflée en $35 \mathrm{~mm}$. C'est ce que l'on 
a appelé le "cinéma-Coutant-Nagra", réalisé avec la caméra autosilencieuse Coutant inventée en 1960 (ou d'autres modèles d'appareils similaires utilisés dans notre pays, l'Arri $B L$, auto-blimpée, ou, un peu plus tard, l'Aaton).

Cinéaste de la spontanéité à ses débuts, Fredi Melchior Murer et les autres (très) jeunes cinéastes de l'underground zurichois, se lancent, eux, avec des budgets dérisoires en $8 \mathrm{~mm}, 16 \mathrm{~mm}$ (très rarement en $35 \mathrm{~mm}$ ). Ils emploient les caméras les plus accessibles financièrement, dont le bruit du moteur (souvent à ressort) n'autorise pas (ou très difficilement) du son direct. Puis le son sera rajouté en post-production (des bruitages et de la musique, plus rarement du texte). C'est le "cinéma-Bolex» très prisé, alors, par les débutants, les expérimentalistes et, aussi, les cinéastes politiques militants (bien sûr, la caméra peut aussi être une Beaulieu, une Canon (plus rare), voire une bonne vieille Arriflex bruyante d'avant l'Arri BL). Pour rappel, la petite et légère (donc très maniable) PaillardBolex (inventée en 1933, puis perfectionnée en 1956), c'est la caméra déjà utilisée en 1961 pour l'œuvre annonciatrice du nouveau cinéma suisse, Quand nous étions petits enfants d'Henry Brandt.

Ces tournages sans son direct obligent à privilégier l'imagination visuelle plutôt que sonore, le lyrisme des images, puisque il n'y a pas de dialogues parlés. Ainsi, contrairement à la vidéo d'aujourd'hui (son et image), cela incite à créer comme au temps du Muet (et, même inconsciemment, comme l'avant-garde des années 1920). A retrouver une écriture qui faisait du Septième art un art spécifique jusqu'à la fin des années 1920, alors que le cinéma sonore (et surtout parlant) deviendra, bien trop fréquemment, un ersatz de la narration littéraire, romanesque ou théâtrale (et ne parlons pas de la télévision: le téléfilm traditionnel repose sur une structure de récit carrément esclave du texte du scénariste). Rejetant les influences de l'Histoire du cinéma, "tuant» les "pères» en opposant individualiste qu'il est, le jeune Murer admire tout de même d'anciens cinéastes privilégiant l'image, comme Chaplin, Flaherty ou le Buñuel des débuts. A sa façon, il réinvente un cinéma indépendant proche de certaines recherches de l'époque où débutait le réalisateur d'Un Chien andalou (Luis Buñuel, Salvador Dali, 1929).

Comme ses confrères de l'underground zurichois, «King Melchior of Cinema» propose, dans ces années de mutation de société, un art très décorseté, comme peuvent l'être la peinture ou la musique improvisée. Il a

«[...] besoin de faire quelque chose dont on ne sait pas exactement ce que cela va donner. Il y a toujours une certaine dose d'aventure 
19 Travelling, $n^{\circ} 27$, Lausanne, déc. 1970-janv. 1971, p. 23.

20 Slogan de Cinéma Marginal Distribution, Lausanne, fin des années 1960.

21 Marcel Leiser, Construire, Zurich, (1969?), sans date, reproduit dans: Marcel Leiser, Films et cinémas suisses: Articles de presse 19641975, chez l'auteur, Lausanne, 1980, p. 198. là-dedans. J'ai toujours conçu mes films d'un point de vue optique. J'ai toujours ces conceptions optiques en tête, et c'est pour moi un bien trop gros effort de les transcrire préalablement sous forme littéraire, c'est-à-dire sous forme de scénario.»19

L'improvisation au tournage prend, en principe, le pas sur les idées initiales. Cette forme d'expression est donc, a priori, incompatible avec un cinéma subventionné qui doit fournir des preuves de contenu préécrites, sur papier, pour exister.

«Faites des films libres, en marge, à petits budgets!»20 A l'instar de moult peintres et autres artistes non reconnus, ces jeunes cinéastes en état d'urgence du tournant des années 1960-1970 veulent d'abord faire des films sans songer forcément à en faire un métier, comme me le confirme ici Köbi Siber, en pleine agit-prop culturelle :

"Comme les jeunes écrivains, ces cinéastes marginaux font ce qu'ils peuvent pour gagner de l'argent. Ils sont graphistes, photographes, commerçants, étudiants, employés postaux auxiliaires. Ils travaillent de temps en temps et s'arrêtent pour faire du cinéma.» $\mathbf{2 1}$

Pour des tournages dans la solidarité-complicité et, si possible, dans le plaisir. Et cela sans salaires ni devant, ni derrière la caméra. Ce «cinémacopain» cadre dans ses objectifs ses proches, les personnages qu'il aime, tandis que le cinéma de système privilégierait, de préférence, le filmage d'acteurs plus ou moins formés et bankables.

A cette époque-là, les gens de caméra (comme les musiciens pop-rock d'ailleurs) prennent fréquemment le pas sur les gens de texte. C'est même devenu une mode. Alors que la création cinématographique se démocratise (pour le meilleur et aussi le pire), les images prennent du pouvoir: elles forgent un environnement artistique qui imprègne la population à plus ou moins forte dose (hélas, la télé va aussi se mettre sérieusement à la tâche, et pas dans le même sens!). L'acceptation du cinéma comme art devient réalité, il n'est plus question de l'appeler «divertissement d'ilotes». En pleins courants énergisants des «sixties", le "cinéma privé» de Murer aura, à sa façon originale, participé à cette libération des images sur grand écran, en produisant des étincelles jubilatoires. Quelles énergies le début du XXI ${ }^{\mathrm{e}}$ siècle devrait-il concevoir pour enfanter, ici, une nouvelle cinématographie libertaire, un mouvement artistique qui aide à mieux respirer, à pleins poumons? Pas facile aujourd'hui, quand il y a davantage de caméras qui contrôlent les citoyens que d'appareils de prises de vues qui rêvent d'utopie. Ou alors, faudrait-il revenir au mutisme, celui, soudain, de Robert Walser, ou celui, permanent, du bouèbe de Höhenfeuer? 